\title{
Autism and psychosis: clinical implications for depression and suicide
}

Rachel Upthegrove MBBS MRCPsych PhD*1,2,3 , Ahmad Abu-Akel PhD ${ }^{4}$, Katharine Chisholm $\mathrm{PhD}^{1}$, Ashleigh Lin $\mathrm{PhD}^{5}$, Sofia Zahid MRes ${ }^{1}$, Mirabel Pelton $\mathrm{MRes}^{1}$, lan Apperly PhD ${ }^{1}$, Peter C Hansen MA (Oxon) DPhil ${ }^{1}$, Stephen J Wood MA (Cantab) PhD ${ }^{1,6,7}$

1. University of Birmingham, School of Psychology, Edgbaston, Birmingham B15 2TT

2. University of Birmingham, Institute of Clinical Sciences, College of Medical and Dental Science, Edgbaston, Birmingham B15 2TT

3. Forward Thinking Birmingham and Birmingham and Solihull Mental Health Foundation Trust, 1 Printing House Street, Birmingham, B4 6DF

4. University of Lausanne, Institute of Psychology, UNIL - Mouline, Géopolis - office 4129 CH-1015 Lausanne, Switzerland

5. Telethon Kids Institute, The University of Western Australia 100 Roberts Rd, Subiaco WA 6008, Australia

6. University of Melbourne, Centre for Youth Mental Health, 35 Poplar Rd, Parkville, VIC 3052

7. Orygen, National Centre of Excellence in Youth Mental Health, 2a/ 2A Hartington Street, Glenroy Victoria 3046

*corresponding author: University of Birmingham, 25 Vincent Drive Edgbaston B16 2NF r.upthegrove@bham.ac.uk 
Abstract:

There is increasing recognition of the co-occurrence of autism and schizophrenia spectrum disorders. However, the clinical significance of this on outcomes such as depression and suicidal thinking has not been explored. This study examines the association of autism spectrum traits, depressive symptoms and suicidal behaviour in individuals with psychotic experiences.

In two cross sectional studies, individuals from a non-help seeking university student sample and patients with first episode psychosis (FEP) service completed standardised measures of autism spectrum traits, psychotic experiences, depressive symptoms and suicidal thinking.

In healthy non-help seeking students, increased autism traits and increased subclinical psychotic experiences were significantly associated with depressive symptoms; a significant interaction effect suggests their combined presence has a greater impact on depression. In FEP, high autism traits and positive symptoms were associated with increased depression, hopelessness and suicidality, however there was no significant interaction effect. In FEP a multiple mediation model revealed that the relationship between autism traits and risk for suicidality was mediated through hopelessness.

Young people with subclinical psychotic experiences and all patients with FEP should be screened for autism spectrum traits, which may have significant impact on clinical outcomes. Tailored interventions for patients with high levels of autistic spectrum comorbidities in FEP should be a priority for future research.

Key words: Autism, Autism Spectrum Disorders, Schizophrenia, Psychosis, Suicide, Depression 


\section{Introduction}

Despite early recognition of similarities between autism and schizophrenia at the times of Bleuler and Kanner, historically these have been understood as two distinct disorders (Kolvin, 1971). Time course (onset in early childhood vs adolescent) and progression (stable vs progressive) are two possible distinguishing features(Chisholm et al., 2015; Wood, 2017). However, it is now recognised that autism spectrum disorder and schizophrenia spectrum disorders share not only some clinical similarities, but also a biological, particularly genetic, liability (Ruzzo and Geschwind, 2016). Whilst autism has long been understood as occurring on a spectrum, with a continuum of severity and deficits identifiable from population to disorder level (Young et al., 2005), recently psychosis has also been suggested to exist on a continuum; positive symptoms are reported in the general population, can be used to identify those at heightened risk of developing psychosis with the ultra-high risk paradigm, and are seen in schizophrenia and other severe mental illnesses (Yung et al., 2006).

At the population level, autistic and psychosis traits are evidentially co-occurring; using the Avon Longitudinal Birth Cohort, Sullivan et al demonstrated that poorer pragmatic language, a key deficit in autism spectrum disorders, was associated with later psychosis and depression (Sullivan et al., 2016). In the same cohort, Siebald and colleagues showed that children aged 8 diagnosed with pervasive developmental disorders, which included the DSM-IV classification of Autism (Association and DSM-IV., 1994), had heightened risk of later psychotic experiences with an odds ratio of 8 (Siebald et al., 2016).

Within clinical populations, the two disorders co-occur at higher rates than would be expected by chance. There is a reported mean incidence of schizophrenia spectrum disorders of $13.8 \%$ in autism spectrum disorder populations, and a mean incidence of autism spectrum disorder of $24.1 \%$ in schizophrenia spectrum disorder populations (Chisholm et al., 2015). Shared clinical features of autism spectrum disorders and schizophrenia spectrum disorders also include difficulty with social communication, emotional expression, salience and restrictive behaviours (Abu-Akel et al., 2017a; Hommer and Swedo, 2015; Ruzzo and Geschwind, 2016). Depression is common in both autism spectrum disorder and schizophrenia spectrum disorders. It has been reported that $14-20 \%$ of young people with autism spectrum disorders have experienced a significant depressive episode by the age of 18 (Gotham et al., 2015). Likewise, up to $80 \%$ of patients with schizophrenia may experience significant depression (Upthegrove 2010), and this has significant long-term consequences for autism spectrum disorders and schizophrenia, including suicide. Lifetime prevalence of completed suicide in schizophrenia is between 710\% (Dutta et al., 2011; Häfner et al., 2005; Upthegrove et al., 2010), and the prevalence of suicidal behaviour in autism spectrum disorder is between 11 and 50\% (Zahid S., 2017).

We have previously shown that depression, positive symptoms and hopelessness are known precursors of self-harm and suicide in schizophrenia spectrum disorders (Upthegrove et al., 
2014). However, the role of autism spectrum disorder traits as a potential pathway to depression and suicidality in schizophrenia spectrum disorders has not been explored to date.

Whilst there has been a considerable amount of recent recognition of the co-occurrence of autism spectrum disorder and schizophrenia spectrum disorders, and how this might inform aetiological understanding, the evidence of the clinical significance of this co-occurrence has yet to develop. This knowledge is needed for evidence-based treatment and management decisions. This study aims to determine the importance, in terms of depression and suicidal behaviour, of autism traits in individuals within the spectrum of psychotic experiences. Given that both disorders are associated with depression and suicidal behaviour, we predict that depression and suicidal behaviour would be increasingly prevalent when autistic spectrum traits and psychosis co-occur.

Hypotheses:

1. Autism traits and subclinical psychotic experiences are associated with increased levels of depressive symptomatology in a healthy, non-help seeking population.

2. Autism traits are associated with positive symptoms and their combined presence increases depression and hopelessness in people with first episode psychosis.

3. Autism traits in people with first episode psychosis increase the risk of suicidality over and above other known risk factors.

\section{Materials and Method:}

\subsection{Sample 1: Healthy, non-help seeking}

Data were collected from 381 University students. Participants were recruited through the University of Birmingham Research Participation Scheme for a course credit or small honorarium. Participants self-reported that they had no history of psychiatric illness, epilepsy, neurological disorders or brain injury (including self-report of any formal diagnosis of ASD, epilepsy, traumatic brain injury, and/or other known neurological or neurodevelopmental condition), and no current or past alcohol and/or substance abuse problems. The University of Birmingham Research Ethics Committee approved the study, and written informed consent was obtained from all participants.

Participants completed the CAPEp (Community Assessment of Psychotic Experiences: positive psychotic-like experiences), which is a 20 -item positive scale questionnaire (Stefanis et al., 2002). The assessment of positive psychotic-like experiences was assessed with the CAPEp rather than the full Community Assessment of Psychotic Experiences (CAPE) as previous literature suggests that negative psychotic experiences maybe cofounded by the presence of autistic traits/autism spectrum disorders (Spek and Wouters, 2010). 
Participants also completed the $A Q$ (The Autism-Spectrum Quotient) (Baron-Cohen et al., 2001), a 50-item questionnaire which examines 5 domains along the autism spectrum: social skills; attention switching; attention to detail; communication; and imagination. Whist the $A Q$ is not a diagnostic instrument, it is a well-validated self-report measure with a Cronbach's Alpha of 0.81 (Hurst et al., 2007). A score of more than 32 is regarded as indicative of autism spectrum disorder and people with autism spectrum disorder rarely score below 26 (Baron-Cohen et al., 2001)

Finally, participants completed the CESD- $R$ (The Center for Epidemiologic Studies Depression Scale-Revised) to assess the presence of depressive symptomatology (Van Dam and Earleywine, 2011).

\subsection{Sample 2: First Episode Psychosis}

Ninety-nine participants with treated first episode psychosis were recruited from Birmingham Early Intervention Services, a community based clinical program with responsibility for the assessment and treatment of patients with first episode psychosis. Birmingham Early Intervention Services see approximately 200 new patients each year, referred from primary and secondary care services. Patients within the service receive antipsychotic medication, care-coordination and psychological interventions as needed.

Ethical approval was given by UK National Research Ethical Committee (NRES), Reference Number 13/WM/0213. Inclusion criteria included age of 16-35 years with a diagnosis of first episode of psychosis in keeping with ICD-10 F20-23, F25-29, F30.2, F31, F32.3. Exclusion criteria included a lack of capacity to consent, unable to communicate verbally in English, and known neurological disorder (including but not limited to epilepsy, traumatic brain injury and learning disability). Patients meeting inclusion and exclusion criteria were approached by their clinical team, and no parameters were set for length of treatment.

Participants completed; SBQ- $R$ (The Suicide Behaviours Questionnaire-Revised) (Osman et al., 2001) which consists of 4 questions that assess different aspects of suicidality. It examines lifetime suicidal ideation and suicide attempt, the frequency of suicidal ideation over the last year, the threat of suicide attempt, and the likelihood of attempting suicide in the future (Osman et al., 2001); AQ (The Autism-Spectrum Quotient) (Baron-Cohen et al., 2001); PANSS (Positive and Negative Syndrome Scale) (Kay et al., 1987), to capture current severity of symptoms on the basis of the standard semi-structured interview. Positive symptoms were assessed by PANSS positive (PANSS-P) subtotal. Depression was rated using PANSS items G1-3 and G6 (PANSS-D), which has been shown to have validity and good correlation with other depression measures used in psychosis (Lako et al., 2012); BHS (Beck Hopelessness Scale) (Beck and Steer, 1988), a 20-item tool designed to measure three major aspects of hopelessness; feelings about the future, loss of motivation, and expectations. 


\subsection{Statistical Analysis}

Inspection of the data suggests that the distribution of all scores in both the healthy and clinical samples were within the accepted range, of -/+ 2 skewness, for parametric analyses (Gravetter and Wallnau, 2014)

\subsubsection{Sample 1:}

The association of the participants' standardised AQ and CAPEp scores and their interaction with the CESD-R scores was examined in a linear regression model using Generalized Linear Models (GLMs), controlling for age and gender. Interactions were probed using the MODPROBE method for SPSS where the effect of one predictor on the outcome measure examined at the mean, 1 s.d. below the mean and 1 s.d. above the mean of the other predictor. This procedure does not involve splitting the sample into smaller groups using these cut-off points, rather it estimates the effect of a predictor on the dependent variable, while holding constant the other predictor at a discrete point (Hayes and Matthes, 2009). Accordingly, this approach allows us to infer from the model the effect of autism traits and positive psychotic-like experiences on depression, in a population with certain composition of positive psychotic-like experiences and autism traits.

\subsubsection{Sample 2: First Episode Psychosis}

In three separate GLMs, linear regression models examined the association of $A Q$ and PANSS-P, and their interaction, with the participants' scores on the PANSS-D, BHS and SBQ$\mathrm{R}$, while controlling for age and gender. However, collinearity diagnostics showed high multicollinearity in all the models for $A Q$ (Variance Inflation Factor (VIF) $=5.91-7.76$ ); PANSS-P (VIF =9.84-16.39); and the interaction term (VIF =19.68-31.30). We addressed the collinearity problem by applying the regression residual method based on the GramSchmidt orthogonalization procedure described in (Draper and Smith, 2014)). A complete orthogonalization (i.e., removal of common variance) of all predictors was accomplished as follows:

Step 1. AQ and PANSSp were orthogonalized by partialling out AQ from PANSS-P.

Step 2. The interaction term ( $A Q \times$ PANSS-P) was orthogonalized by partialling out both $A Q$ and PANSS-P.

This procedure rendered 3 uncorrelated variables: AQ, PANSS-P residual, and AQ $\times$ PANSS-P residual. The standardized values of these orthogonalized predictors were used in the subsequent GLMs.

Additionally, to examine whether the effect of $A Q$ on suicidality was mediated by positive symptoms, hopelessness, and/or depression, a multiple mediation analysis was conducted 
using SPSS Macro for Multiple Mediation (Preacher and Hayes, 2008). This analysis was conducted with 5000 bootstrap resamples, while controlling for age and gender.

\section{Results:}

3.1 Sample 1: Healthy non help-seeking: The sample was $21 \%$ male (male/female=80/301); Age range 17-39; mean age $(S D)=20.61$ (3). Mean AQ score was 15.32 (6.04). Two participants (0.52\%) had a score indicative of likely autism spectrum disorder (as defined by $A Q>32$ )(Baron-Cohen et al., 2001), and 19 (4.99\%) when using the more lenient indicative score of AQ>26 (Woodbury-Smith et al., 2005). Mean CAPEp and CESD-R scores were 26.55 (4.24) and 12.56 (10.81), respectively. See table 1.

\subsection{Sample 2: First Episode Psychosis: The FEP sample was $67.7 \%$ male} (male/female=67/32); Age range 16-36, mean age $(S D)=25.61$ (5.0). Mean AQ score was 20.76 (7.96). Twenty-five participants (25\%) scored $>26$ or above on the $A Q$, of which 8 participants (9.2\%) scored over 32. One per cent of participants had a lifetime history of suicide plans (with or without attempt), and $24 \%$ a lifetime history of suicide attempt.

\section{***Table 1 about here ***}

3.3 Hypotheses 1: Autism traits and psychosis are associated with increased incidence of depression in a healthy, non-help seeking population

The GLM regression model yielded an overall significant model (Wald $\mathrm{x}_{\mathrm{x}}=92.23, \mathrm{df}=5$; $p<0.001 ; R^{2}=0.216$ ). As can be seen from Table 2 , the analysis revealed a significant positive association between CESD-R and the interaction term of AQ $\times$ CAPEp. $15.6 \%$ of the total variance explained by the model was accounted for by the interaction term $\left(R^{2}\right.$ change $=0.034, F=16.03 ; p<0.001)$.

\footnotetext{
***Table 2 about here ${ }^{* * *}$
}

The analysis presented in Figure 1 probes the interaction between $A Q$ and CAPEp. Figure $1 A$ shows that while AQ had no effect on CESD-R at 1SD below the mean of CAPEp ( $\beta$ (SE) $=0.03$ (.73), $t=0.043, p=0.97)$, it was significantly associated with higher CESD-R scores at the mean $(\beta(\mathrm{SE})=2.11(0.53), t=4.01, p<0.001)$ and $1 \mathrm{SD}$ above the mean $(\beta(\mathrm{SE})=4.20(0.76)$, $t=5.54, p<0.001$ ) of CAPEp. Conversely, in Figure 1B, the association between CAPEp and 
CESD-R was not significant at 1SD below the mean $(\beta(S E)=0.50(0.87), t=0.57, p=0.57)$ of $A Q$, but positively significant at the mean $(\beta(S E)=2.58(0.55), t=4.66, p<0.001)$ and at $1 \mathrm{SD}$ above the mean $(\beta(S E)=4.66(0.63), t=7.40, p<0.001)$ of $A Q$. See also supplementary Figure 1.

***figure 1 about here***

3.4 Hypotheses 2: Autism traits are associated with positive symptoms and their combined presence increases depression and hopelessness in FEP.

There was a significant correlation between PANSS-P and AQ total scores $(r=0.42, p<0.001)$.

\subsubsection{Depression:}

The GLM results yielded an overall significant model $\left(\right.$ Wald $_{x 2}=30.86, d f=5 ; p<0.001$;

$\left.R^{2}=0.300\right)$. As can be seen from Table 3, there was a significant positive association between PANSS-D scores and both AQ and PANSS-P scores. The interaction term of AQ $\times$ PANSS-P was non-significant.

\subsubsection{Hopelessness:}

The GLM results yielded an overall significant model ( Wald $_{x 2}=28.92, d f=5 ; p<.001 ; R^{2}=.323$ ). As can be seen from Table 3, there was a significant positive association between BHS total scores and both AQ and PANSS-P scores. The interaction term of AQ x PANSS-P was nonsignificant.

\section{***table 3 about here ${ }^{* * *}$}

3.5 Hypotheses 3: Autism traits in first episode psychosis increase risk of suicidality over and above other known risk factors

The GLM results yielded an overall significant model ( Wald $_{x 2}=16.47, d f=5 ; p=.006 ; R^{2}=.200$ ). As can be seen from Table 3, there was a significant positive association between SBQ- $R$ total scores and both $A Q$ and PANSS-P scores. There was also a significant association between gender and $S B Q-R$, such that males $(M(s e)=2.61(0.10))$ reported significantly lower $S B Q-R$ scores compared to the females $(M(s e)=2.94(0.12))$. The interaction term of $A Q x$ PANSS-P was non-significant. 
Multiple mediation model: The overall multiple mediation model was significant $(F(6,65)=$ $\left.7.22, p<0.001, R^{2}=0.40\right)$. There was a significant indirect effect of $A Q$ through the mediator hopelessness on SBQ-R ( $a b=0.117$, bias-corrected $95 \% \mathrm{Cl}=0.05-0.22)$, accounting for $71 \%$ of the total effect of AQ on SBQ-R (c path $=0.164(0.057), t=2.88, p=.005$ ). The indirect effects of PANSS-D ( $a b=0.057$, bias-corrected $95 \% \mathrm{Cl}=-0.007-0.139)$ and PANSS-P $(a b=$ 0.019 , bias-corrected $95 \% \mathrm{Cl}=-0.043-0.095$ ) were non-significant (see Figure 2).

$* * *$ figure 2 about here***

\section{Discussion:}

The aim of this study was to determine the importance of autism traits in individuals within the spectrum of psychotic experiences in the presence of depression and suicidal behaviour. The data presented from the FEP sample demonstrated that the prevalence of autistic spectrum traits is high, with $25 \%$ of the sample having $A Q$ score indicative of need for formal diagnostic assessment and in keeping with the majority of studies reporting this cooccurrence (Chisholm et al., 2015). Our novel findings are that this co-occurrence is clinically important; autism traits and positive symptoms were associated with a significantly increased level of depressive symptoms, hopelessness and suicidal thinking. Within the nonhelp seeking population, autism traits and psychosis traits were associated with likewise higher levels of depressive symptomatology, such that their combined effect was greatest when both autism and psychosis traits are high. These findings suggest that autism traits are associated with increased depressive symptoms across the psychosis spectrum.

Our findings are in keeping with the multiple aetiology and increased vulnerability models of autism spectrum disorder and psychosis co-occurrence (Chisholm et al., 2015). Although it is speculative to draw firm conclusions from our cross-sectional studies, with a high proportion of females in our university educated, healthy non-help seeking sample, it is possible that there is an increased vulnerability for both traits through a shared genetic liability (Carroll and Owen, 2009). It is also possible that the perception of genetic liability for both disorders leads to further stigmatizing attitudes, and subsequent depressive symptoms (Serafini et al., 2011). It is likewise speculative to draw inference on a direction of causality in the vulnerability to mood disorders and suicide autism spectrum traits and psychosis may bring. However, as autism traits are suggestive of long-term deficits, it is possible that these deficits lead to an increase to both psychotic-like symptoms and depression. Recent evidence suggests that anomalous experiences such as hallucinations are common and associated with significant distress in adults with autism spectrum disorder (Milne et al., 2017). The precipitation of psychotic symptoms may be via a reciprocal pathway of stress vulnerability and depression (Myin-Germeys and van Os, 2007), and autism spectrum disorder traits add to this vulnerability. Freeman et al argue that emotion deregulation is responsible for the generation of clinically significant positive symptoms in 
patients who cannot manage their emotional distress (Freeman et al., 2001). Thus, we may see higher rates of emotional dysregulation in patients with psychosis and high autism traits through this route. This is in keeping with an 'autistic pathway' to psychosis, mediated by emotional deregulations as Toal et al propose (Toal et al., 2009).

Craddock and Owen describe a continuum model of psychosis and autism spectrum disorder traits with developmental disorders and autism traits at one end, through to schizophrenia, schizoaffective disorder and bipolar disorder with increasing frequency of affective symptoms at the other end (Craddock and Owen, 2010). Our results would challenge this model as the combined presence of autism traits and positive symptoms gives an increase association with depression with complex interaction. Our results would not support a diametric model of autism spectrum disorders and psychosis, which would predict that the combined presence of both would result in lesser impact. However, it is possible that their diametric effects are specific to the cognitive, but not an affective domain (Abu-Akel et al., 2017a). This is consistent with a recent study of college students showing that the cooccurrence of autism and schizotypal traits was associated with better social and executive function but poorer emotional expression (Shi et al., 2017).

We reported that an interactive effect of autism traits and positive symptoms on depression was not seen in first episode psychosis, rather both had independent effects. Autism traits appear to increase risk of suicidal behaviour through increased hopelessness in first episode psychosis, even after controlling for the influence of age and gender. Once a level of illness severity reaches diagnostic significance in the psychosis spectrum, the role of other mediators such as stigma, shame, and powerlessness influence hopelessness and suicidal thinking (Upthegrove et al., 2014). Results from the present study also suggest a role for autism spectrum disorder traits. Matsuo (Matsuo et al., 2015) demonstrated that nearly $50 \%$ of patients with schizophrenia had autism traits at levels typical for sub-threshold or threshold autism spectrum disorder, and that there was no difference between remitted or unremitted subjects. Thus, the indication, when combined with our novel findings, is that autism spectrum disorder traits can be identified at any stage or state of psychosis, and are likely to increase mood symptoms, hopelessness and suicidality.

Dutta and Reininghaus show the risk for suicide within psychosis lasts for 15 or more years after diagnosis (Dutta et al., 2011; Reininghaus et al., 2015). Our findings of the role of autism spectrum disorder traits in depressive symptoms and suicidality are both novel and significant; it is possible that autism spectrum disorder traits contribute to a longer-term risk. It is speculative to consider how autism spectrum disorder traits might influence suicidal thinking in psychosis; it is possible they add to the isolation and social impact seen in early psychosis. Emotional impairment, anhedonia, alexithymia (the inability to identify one's feelings), social isolation and social withdrawal are common experiences in both autism and psychosis (Larson et al., 2016). The combined presence of psychosis and autism traits may contribute to impairment in emotional communication, leading to such factors 
that increase risk of depression and hopelessness such as isolation. Eack and colleagues demonstrate clear difficulties in facial emotional perception in autism, and Mehta et al show difficulties in social interaction in schizophrenia (Eack et al., 2014; Mehta et al., 2014); when these are present, this may lead to further social isolation. Within populations with psychosis, it has also been suggested that autism traits may also bring an additional impact on theory of mind deficits (Pickup and Frith, 2001), resulting in further social isolation. Some evidence suggests that this is specific to affective rather than cognitive theory of mind (Shamay-Tsoory and Aharon-Peretz, 2007).

In this paper, we have assessed two samples across the spectrum of psychosis experiences; a healthy non-help seeking population and full first episode psychosis. Relatively large sample sizes allowed for regression analyses using linear models while controlling for age and gender. However, the study does have limitations that need to be acknowledged. Our non-help seeking sample was drawn from university students, with a high proportion of female participants and thus is not readily generalizable to the population as a whole, and is likely to be significantly higher in educational attainment than a general population. We used a self-report for health status in this group and a structured neurodevelopmental history has not been taken. Within the FEP sample, depressive symptomatology was measured by PANSS-D and not a more specific scale for the assessment of depression in schizophrenia, and thus may have been influenced by the presence of participants with significant negative symptoms. PANNS- Positive symptoms were strongly correlated with AQ score, and although there was not an interaction effect, suggesting independent effects, it is possible some autistic features measured were secondary to positive symptoms or disorganization. Suicidal behavior was recorded from self-report, and whilst the SBQ-R contains a measure that of past lifetime history of suicidal behavior, we were not able to control for past suicide attempts which will have been variously proximal to our assessment time. A further limitation is the self-report AQ rather than diagnostic interview in both samples, which further leads to the exploratory nature of our results. However the AQ has previously been used to assess traits with validity and in clinical populations (Milne et al., 2017). At present, there is not a valid diagnostic measure for autism in schizophrenia, and this is clearly an area of future research need. As a cross-sectional study, with autism traits measured by self-report, limited speculation is possible in direction of influence.

\section{Conclusion:}

Our results suggest that when autism spectrum traits and psychotic symptoms co-occur, there are significant implications for depressive psychopathology and suicidal behaviour. Clinical services for psychosis should be vigilant in detecting autism traits, as this may give additional information for prediction of depression, hopelessness and risk of suicide. The interventions routinely offered for first episode psychosis, including Cognitive Behavioural Therapy for psychosis and Behavioural Family Therapy, may also need to be tailored for patients with significant autism spectrum disorder traits, to ensure they are accessible and effective. As this group is at heightened risk for depression, hopelessness and suicide, these interventions are indeed needed. 


\section{References:}

Abu-Akel, A., Apperly, I.A., Wood, S.J., Hansen, P.C., Mevorach, C., 2017a. Autism tendencies and psychosis proneness interactively modulate saliency cost. Schizophrenia bulletin 43(1), 142-151. Abu-Akel, A.M., Apperly, I.A., Wood, S.J., Hansen, P.C., 2017b. Autism and psychosis expressions diametrically modulate the right temporo-parietal junction. Social neuroscience 12, 506-518. Association, A.P., DSM-IV., A.P.A.T.F.o., 1994. Diagnostic and statistical manual of mental disorders: DSM-IV. Amer Psychiatric Pub Inc.

Baron-Cohen, S., Wheelwright, S., Skinner, R., Martin, J., Clubley, E., 2001. The autism-spectrum quotient ( $A Q$ ): Evidence from asperger syndrome/high-functioning autism, malesand females, scientists and mathematicians. Journal of autism and developmental disorders 31(1), 5-17.

Beck, A.T., Steer, R.A., 1988. Beck hopelessness scale. Psychological Corporation San Antonio, TX. Carroll, L.S., Owen, M.J., 2009. Genetic overlap between autism, schizophrenia and bipolar disorder. Genome medicine 1(10), 102.

Chisholm, K., Lin, A., Abu-Akel, A., Wood, S.J., 2015. The association between autism and schizophrenia spectrum disorders: a review of eight alternate models of co-occurrence.

Neuroscience \& Biobehavioral Reviews 55, 173-183.

Craddock, N., Owen, M.J., 2010. The Kraepelinian dichotomy - going, going... but still not gone. The British Journal of Psychiatry 196(2), 92-95.

Draper, N.R., Smith, H., 2014. Applied regression analysis. John Wiley \& Sons.

Dutta, R., Murray, R.M., Allardyce, J., Jones, P.B., Boydell, J., 2011. Early risk factors for suicide in an epidemiological first episode psychosis cohort. Schizophrenia Research 126(1), 11-19.

Eack, S.M., Mazefsky, C.A., Minshew, N.J., 2014. Misinterpretation of facial expressions of emotion in verbal adults with autism spectrum disorder. Autism 1, 8.

Freeman, D., Garety, P.A., Kuipers, E., 2001. Persecutory delusions: developing the understanding of belief maintenance and emotional distress. Psychological medicine 31(7), 1293-1306.

Gotham, K., Brunwasser, S., Lord, C., 2015. Depressive and anxiety symptom trajectories from school age through young adulthood in samples with autism spectrum disorder and developmental delay. Journal of the American Academy of Child and Adolescent Psychiatry 54(5), 369-376. e363. Gravetter, F., Wallnau, L., 2014. Introduction to the t statistic. Essentials of Statistics for the Behavioral Sciences 8, 252.

Häfner, H., Maurer, K., Trendler, G., an, d., Heiden,Wolfram, Schmidt, M., 2005. The early course of schizophrenia and depression*. European archives of psychiatry and clinical neuroscience 255(3), 167-173.

Hayes, A.F., Matthes, J., 2009. Computational procedures for probing interactions in OLS and logistic regression: SPSS and SAS implementations. Behavior research methods 41(3), 924-936.

Hommer, R.E., Swedo, S.E., 2015. Schizophrenia and autism-related disorders. Schizophrenia bulletin 41(2), 313-314.

Hurst, R., Mitchell, J., Kimbrel, N.A., Kwapil, T., Nelson-Gray, R., 2007. Examination of the reliability and factor structure of the Autism Spectrum Quotient $(A Q)$ in a non-clinical sample. Personality and Individual Differences 43(7), 1938-1949.

Kay, S.R., Flszbein, A., Opfer, L.A., 1987. The positive and negative syndrome scale (PANSS) for schizophrenia. Schizophrenia bulletin 13(2), 261.

Kerns, C.M., Kendall, P.C., Zickgraf, H., Franklin, M.E., Miller, J., Herrington, J., 2015. Not to be overshadowed or overlooked: functional impairments associated with comorbid anxiety disorders in youth with ASD. Behavior therapy 46(1), 29-39.

Kolvin, I., 1971. Studies in the childhood psychoses: I. Diagnostic criteria and classification. The British Journal of Psychiatry.

Lako, I.M., Bruggeman, R., Knegtering, H., Wiersma, D., Schoevers, R., Slooff, C., Taxis, K., 2012. A systematic review of instruments to measure depressive symptoms in patients with schizophrenia. Journal of affective disorders 140(1), 38-47. 
Larson, F.V., Wagner, A.P., Jones, P.B., Tantam, D., Lai, M.-C., Baron-Cohen, S., Holland, A.J., 2016. Psychosis in autism: comparison of the features of both conditions in a dually affected cohort. The British Journal of Psychiatry, bjp. bp. 116.187682.

Matsuo, J., Kamio, Y., Takahashi, H., Ota, M., Teraishi, T., Hori, H., Nagashima, A., Takei, R., Higuchi, T., Motohashi, N., 2015. Autistic-like traits in adult patients with mood disorders and schizophrenia. PloS one 10(4), e0122711.

Mehta, U.M., Thirthalli, J., Bhagyavathi, H., Kumar, J.K., Subbakrishna, D., Gangadhar, B.N., Eack, S.M., Keshavan, M.S., 2014. Similar and contrasting dimensions of social cognition in schizophrenia and healthy subjects. Schizophrenia research 157(1), 70-77.

Michail, M., Birchwood, M., 2014. Social anxiety in first-episode psychosis: the role of childhood trauma and adult attachment. Journal of affective disorders 163, 102-109.

Milne, E., Dickinson, A., Smith, R., 2017. Adults with autism spectrum conditions experience increased levels of anomalous perception. PLOS ONE 12(5), e0177804.

Myin-Germeys, I., van Os, J., 2007. Stress-reactivity in psychosis: Evidence for an affective pathway to psychosis. Clinical Psychology Review 27(4), 409-424.

Osman, A., Bagge, C.L., Gutierrez, P.M., Konick, L.C., Kopper, B.A., Barrios, F.X., 2001. The Suicidal Behaviors Questionnaire-Revised (SBQ-R): validation with clinical and nonclinical samples.

Assessment 8(4), 443-454.

Pickup, G.J., Frith, C.D., 2001. Theory of mind impairments in schizophrenia: symptomatology, severity and specificity. Psychological medicine 31(02), 207-220.

Preacher, K.J., Hayes, A.F., 2008. Asymptotic and resampling strategies for assessing and comparing indirect effects in multiple mediator models. Behavior research methods 40(3), 879-891.

Reininghaus, U., Dutta, R., Dazzan, P., Doody, G.A., Fearon, P., Lappin, J., Heslin, M., Onyejiaka, A., Donoghue, K., Lomas, B., 2015. Mortality in Schizophrenia and Other Psychoses: A 10-Year Follow-up of the $\mathbb{E}$ SOP First-Episode Cohort. Schizophrenia bulletin 41(3), 664-673.

Ruzzo, E.K., Geschwind, D.H., 2016. Schizophrenia genetics complements its mechanistic understanding. Nature neuroscience.

Serafini, G., Pompili, M., Haghighat, R., Pucci, D., Pastina, M., Lester, D., Angeletti, G., Tatarelli, R., Girardi, P., 2011. Stigmatization of schizophrenia as perceived by nurses, medical doctors, medical students and patients. Journal of psychiatric and mental health nursing 18(7), 576-585.

Shamay-Tsoory, S.G., Aharon-Peretz, J., 2007. Dissociable prefrontal networks for cognitive and affective theory of mind: a lesion study. Neuropsychologia 45(13), 3054-3067.

Shi, L.-j., Liu, W.-h., Shi, H.-S., Yan, C., Wang, Y., Wang, Y., Cheung, E.F., Chan, R.C., 2017. Cooccurrence of autistic and schizotypal traits and its association with emotional and psychosocial function in Chinese college students. Psychiatry Research 248, 64-70.

Siebald, C., Khandaker, G.M., Zammit, S., Lewis, G., Jones, P.B., 2016. Association between childhood psychiatric disorders and psychotic experiences in adolescence: A population-based longitudinal study. Comprehensive Psychiatry 69, 45-52.

Spek, A.A., Wouters, S.G., 2010. Autism and schizophrenia in high functioning adults: Behavioral differences and overlap. Research in Autism Spectrum Disorders 4(4), 709-717.

Stefanis, N., Hanssen, M., Smirnis, N., Avramopoulos, D., Evdokimidis, I., Stefanis, C., Verdoux, H., Van Os, J., 2002. Evidence that three dimensions of psychosis have a distribution in the general population. Psychological medicine 32(02), 347-358.

Sullivan, S.A., Hollen, L., Wren, Y., Thompson, A.D., Lewis, G., Zammit, S., 2016. A longitudinal investigation of childhood communication ability and adolescent psychotic experiences in a community sample. Schizophrenia research 173(1), 54-61.

Toal, F., Bloemen, O.J., Deeley, Q., Tunstall, N., Daly, E.M., Page, L., Brammer, M.J., Murphy, K.C., Murphy, D.G., 2009. Psychosis and autism: magnetic resonance imaging study of brain anatomy. The British Journal of Psychiatry 194(5), 418-425.

Upthegrove, R., Birchwood, M., Ross, K., Brunett, K., McCollum, R., Jones, L., 2010. The evolution of depression and suicidality in first episode psychosis. Acta Psychiatrica Scandinavica 122(3), 211-218. 
Upthegrove, R., Ross, K., Brunet, K., McCollum, R., Jones, L., 2014. Depression in first episode psychosis: the role of subordination and shame. Psychiatry Res 217(3), 177-184.

Van Dam, N.T., Earleywine, M., 2011. Validation of the Center for Epidemiologic Studies Depression Scale-Revised (CESD-R): Pragmatic depression assessment in the general population. Psychiatry research 186(1), 128-132.

Wood, S.J., 2017. Autism and schizophrenia: one, two or many disorders? RCP.

Woodbury-Smith, M.R., Robinson, J., Wheelwright, S., Baron-Cohen, S., 2005. Screening adults for Asperger syndrome using the $A Q$ : A preliminary study of its diagnostic validity in clinical practice. Journal of autism and developmental disorders 35(3), 331-335.

Young, D., Murray, V., McKee, I., Miller, P.M., Muir, W.J., Pelosi, A.J., Blackwood, D.H.R., 2005.

Dimensions and classes of psychosis in a population cohort: a four class, four dimension model of schizophrenia and affective psychoses.

Yung, A.R., Stanford, C., Cosgrave, E., Killackey, E., Phillips, L., Nelson, B., McGorry, P.D., 2006.

Testing the Ultra High Risk (prodromal) criteria for the prediction of psychosis in a clinical sample of young people. Schizophrenia Research 84(1), 57-66.

Zahid S., U.R., 2017. Suicidality in Autistic Spectrum Disorders: a systematic review. Crisis. 
Table 1. Clinical descriptors of Sample 1 and 2

\begin{tabular}{|l|l|l|l|}
\hline Sample 1 & Minimum & Maximum & Mean (s.d.) \\
\hline Age & 17 & 39 & $20.61(3)$ \\
\hline AQ & 3 & 35 & $15.3(6.0)$ \\
\hline CAPEp & 20 & & \\
\hline CESD-R & 0 & 40 & $26.55(4.24)$ \\
\hline & & 73 & $12.56(10.81)$ \\
\hline Sample 2 & Minimum & Maximum & Mean (s.d.) \\
\hline Age & 16 & 36 & 25.61 (5) \\
\hline AQ Total Score & 5.00 & 42.00 & $20.76(7.96)$ \\
\hline PANSS Negative & 7.00 & 31.00 & $12.36(5.29)$ \\
\hline PANSS Positive & 7.00 & 30.00 & $12.34(5.65)$ \\
\hline PANSS-D & 4 & 22.00 & $8.54(4.31)$ \\
\hline BHS Total & 0 & 20.00 & $6.36(5.43)$ \\
\hline SBQ-R Total & 3.00 & 17.00 & $7.45(4.07)$ \\
\hline
\end{tabular}

Table 2. Summary of regression coefficients with total CESD-R scores as the dependent variable in healthy, non-help seeking sample

\begin{tabular}{|l|l|l|l|l|}
\hline & $\boldsymbol{\beta}$ & SE (95\% C.I.) & Wald $_{\mathbf{x} 2}$ & $\mathbf{p}$ \\
\hline Age & -0.481 & $0.152(-0.778,-0.183)$ & 10.04 & $\mathbf{0 . 0 0 2}$ \\
\hline Gender = Male & -0.272 & $1.238(-2.698,2.154)$ & .05 & 0.83 \\
\hline AQ & 2.118 & $0.523(1.093,3.142)$ & 16.40 & $<0.001$ \\
\hline CAPEp & 2.585 & $0.548(1.511,3.659)$ & 22.24 & $<0.001$ \\
\hline AQ $\times$ CAPEp & 2.081 & $0.516(1.071,3.091)$ & 16.30 & $<0.001$ \\
\hline
\end{tabular}


Table 3. Summary of regression coefficients of three models with total PANSS-D score, BHS total and SBQ-R as dependent variables in first-episode psychosis sample

\begin{tabular}{|c|c|c|c|c|}
\hline Depression (PANSS-D) & $\beta$ & SE (95\% C.I.) & Wald $_{x 2}$ & $p$ \\
\hline Age & 0.019 & $0.013(-0.006,0.044)$ & 2.19 & 0.139 \\
\hline Gender $=$ Male & -0.109 & $0.141(-0.386,0.167)$ & 0.60 & 0.44 \\
\hline$A Q$ & 0.305 & $0.068(0.171,0.438)$ & 20.05 & $<0.001$ \\
\hline PANSS-Positive & 0.238 & $0.059(0.121,0.354)$ & 16.07 & $<0.001$ \\
\hline AQ $\times$ PANSS-Positive & -0.047 & $0.060(-0.165,0.071)$ & 0.61 & 0.44 \\
\hline \multicolumn{5}{|c|}{ Hopelessness (BHS Total) } \\
\hline Age & 0.013 & $0.021(-0.029,0.054)$ & 0.34 & 0.56 \\
\hline Gender $=$ Male & -0.054 & $0.236(-0.517,0.408)$ & 0.05 & 0.82 \\
\hline$A Q$ & 0.616 & $0.120(0.380,0.851)$ & 26.28 & $<0.001$ \\
\hline PANSS-Positive & 0.193 & $0.098(0.001,0.385)$ & 3.88 & 0.049 \\
\hline AQ $\times$ PANSS-Positive & -0.082 & $0.142(-0.361,0.197)$ & .33 & 0.57 \\
\hline \multicolumn{5}{|l|}{ Suicidality (SBQ-R) } \\
\hline Age & -0.003 & $0.015(-0.032,0.027)$ & 0.03 & 0.87 \\
\hline Gender $=$ Male & -0.321 & $0.156(-0.626,-0.016)$ & 4.23 & 0.039 \\
\hline$A Q$ & 0.235 & $0.079(0.082,0.389)$ & 8.99 & 0.003 \\
\hline PANSS-Positive & 0.190 & $0.076(0.041,0.339)$ & 6.21 & 0.013 \\
\hline$A Q \times$ PANSS-Positive & 0.017 & $0.103(-0.184,0.219)$ & 0.03 & 0.87 \\
\hline
\end{tabular}


Fig. 1. The interactive effect of autism traits and positive psychotic-like experiences on depressive symptoms.
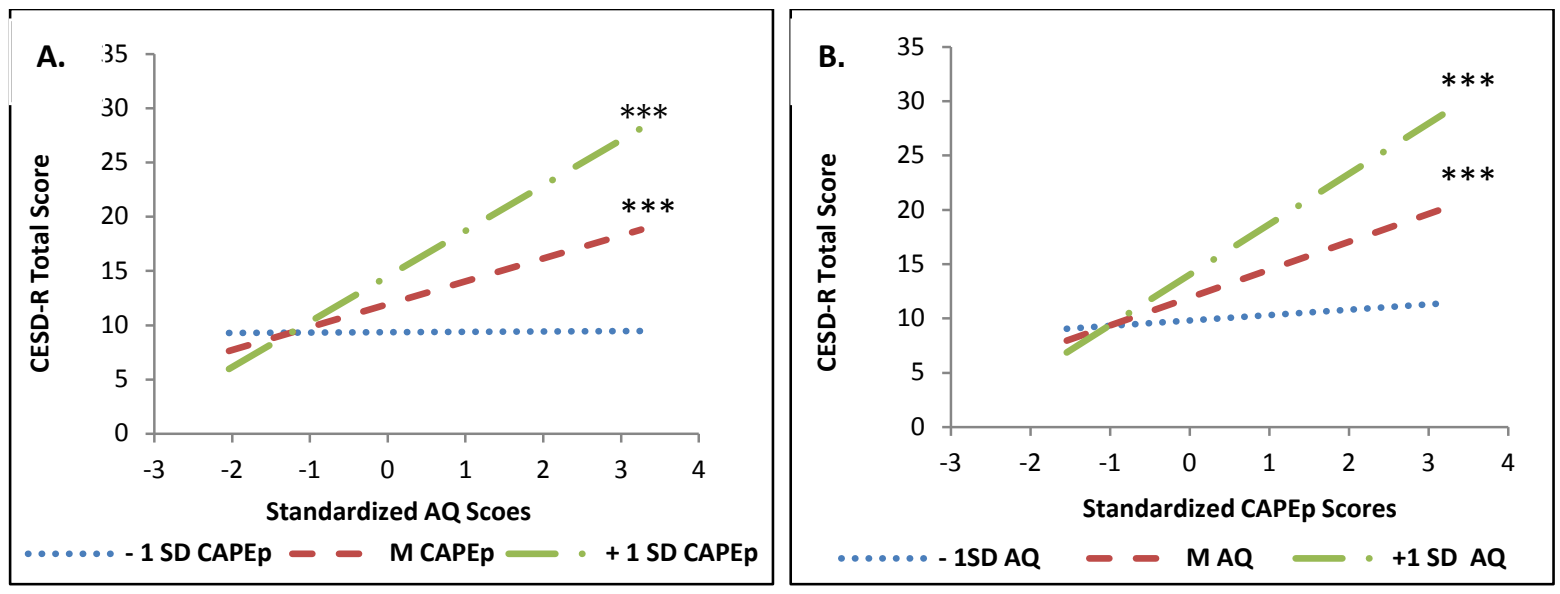

(A) Visualizes the association between autism traits and depressive symptoms by plots of simple regression lines with low ( -1 SD), average, and high (+1 SD) CAPEp scores as moderators, showing an increase in the positive effect of autism traits in individuals scoring average and above on CAPEp. (B) Visualizes the association between subclinical psychotic experiences and depressive symptoms by plots of simple regression lines with low (-1 SD), average, and high AQ (+1 SD), showing a positive effect of positive subclinical psychotic experiences in individuals scoring average and above on $A Q$.

$* * *=p$-value $<.001 . \mathrm{AQ}=$ Autism Quotient $\mathrm{CAPEp}=$ Community Assessment of Psychic Experiences, positive subscale; CESD-R= The Center for Epidemiologic Studies Depression Scale-Revised (Fig. 1S. depicts the raw data of the model presented in Fig. 1A and 1B). 
Fig. 2. Multiple mediation model testing the effect of autism traits on suicidality through the mediators: hopelessness, PANSS positive and PANSS depressive symptoms.

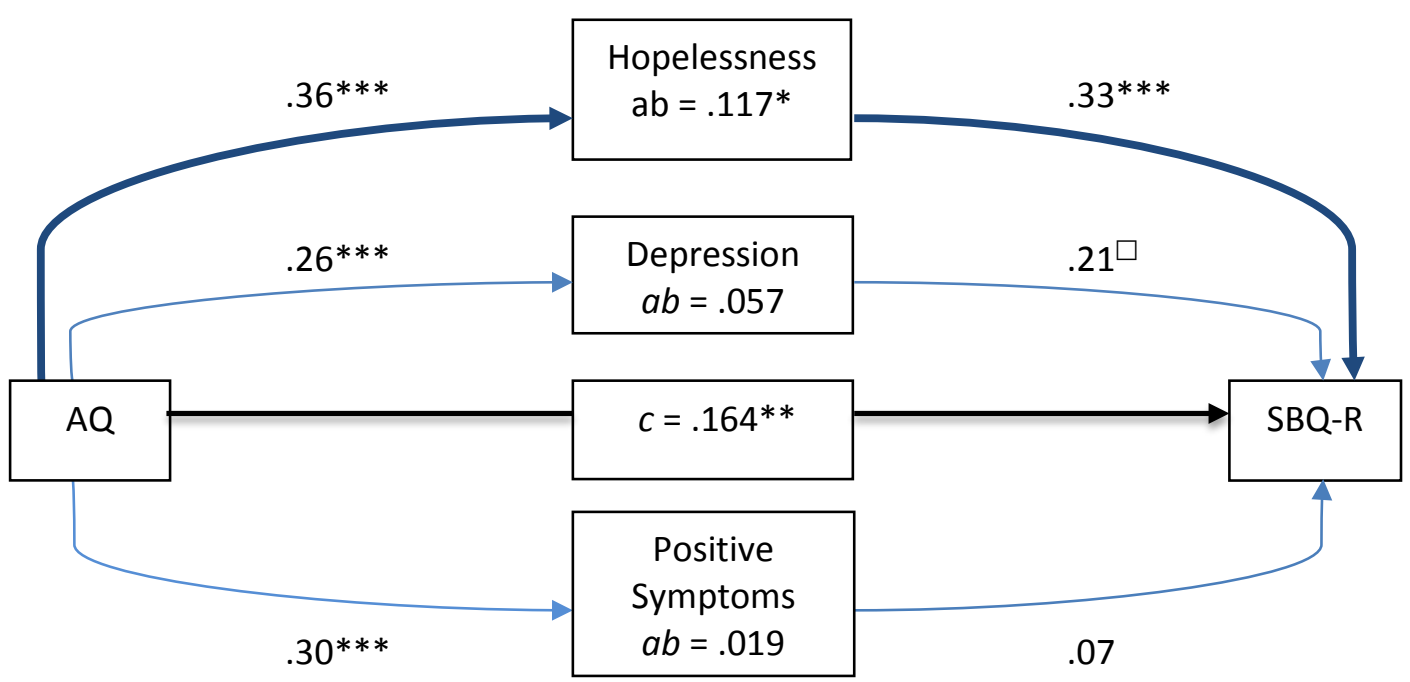

$a b=$ coefficients of the 5000 bootstrap resamples indirect effects of AQ on SBQ-R through the proposed mediators, while controlling for age and gender. $c$ represents the coefficient of the direct effect of $A Q$ on SBQ-R; $A Q=$ Autism Quotient; PANSS= Positive and Negative Syndrome Scale; SBQ-R= The Suicide Behaviours questionnaire-revised; ${ }^{*}=p<.05 ;{ }^{* *} p<.01$; $* * * p<.001 ; * p=.078$ 
Figure 1S: 3-D representation of the relationship of autism traits (AQ) and positive psychotic-

like experiences (CAPEp) (represented as standardized Z scores) with depressive symptomatology

(CESD-R). The negative scores represent low tendencies and the positive scores represent high tendencies.

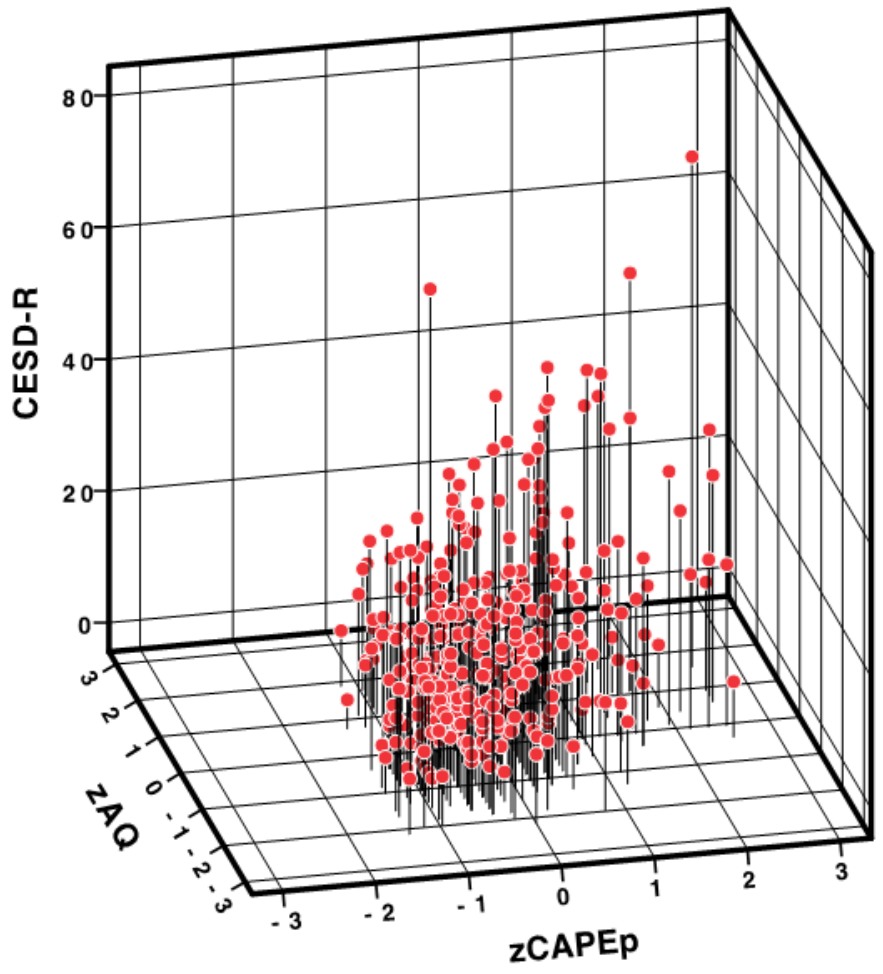

